\title{
CRIM-TRACK: Sensor system for detection of criminal chemical substances
}

\author{
Jens K. Munk*a, Ole T. Buus ${ }^{\mathrm{b}}$, Jan Larsen ${ }^{\mathrm{b}}$, Eleftheria Dossic, Sol Tatlow ${ }^{\mathrm{d}}$, Lina Lässige, Lars \\ Sandström ${ }^{\mathrm{f}}$, Mogens H. Jakobsen ${ }^{\mathrm{a}}$ \\ ${ }^{a}$ Dept. of Micro- and Nanotechnology, Technical University of Denmark, Ørsteds Plads, 2800 Kgs. \\ Lyngby, Denmark; ${ }^{b}$ Dept. of Applied Mathematics and Computer Science, Technical University of \\ Denmark, Richard Petersens Plads, 2800 Kgs. Lyngby, Denmark; ${ }^{\mathrm{c} C e n t r e ~ f o r ~ D e f e n c e ~ C h e m i s t r y, ~}$ \\ Cranfield University, Defence Academy of United Kingdom, Shrivenham, SN6 8LA, United

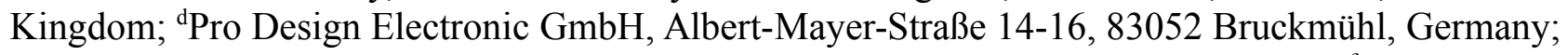 \\ ${ }^{\mathrm{e}}$ Securetec Detektions-Systeme AG, Lilienthalstrasse 7, 85579 Neubiberg, Germany; ${ }^{\mathrm{f}}$ Gammadata \\ Instrument AB, Vallongatan 1, 75228 Uppsala, Sweden
}

\begin{abstract}
Detection of illegal compounds requires a reliable, selective and sensitive detection device. The successful device features automated target acquisition, identification and signal processing. It is portable, fast, user friendly, sensitive, specific, and cost efficient. LEAs are in need of such technology. CRIM-TRACK is developing a sensing device based on these requirements. We engage highly skilled specialists from research institutions, industry, SMEs and LEAs and rely on a team of end users to benefit maximally from our prototypes. Currently we can detect minute quantities of drugs, explosives and precursors thereof in laboratory settings. Using colorimetric technology we have developed prototypes that employ disposable sensing chips. Ease of operation and intuitive sensor response are highly prioritized features that we implement as we gather data to feed into machine learning. With machine learning our ability to detect threat compounds amidst harmless substances improves. Different end users prefer their equipment optimized for their specific field. In an explosives-detecting scenario, the end user may prefer false positives over false negatives, while the opposite may be true in a drug-detecting scenario. Such decisions will be programmed to match user preference. Sensor output can be as detailed as the sensor allows. The user can be informed of the statistics behind the detection, identities of all detected substances, and quantities thereof. The response can also be simplified to "yes" vs. "no". The technology under development in CRIM-TRACK will provide custom officers, police and other authorities with an effective tool to control trafficking of illegal drugs and drug precursors.
\end{abstract}

Keywords: Trace vapor sensing, drugs, explosives, precursors, colorimetry, optics, disposable chip, user friendly.

\section{INTRODUCTION}

The aim of the CRIM-TRACK project is to demonstrate a working sensing device that can be developed into a portable, miniaturized, automated, rapid, low cost, highly sensitive, and simple "sniffer" and detection unit, based on a disposable micro-colorimetric chip. The unit can be used for identification of a wide variety of illegal drugs, drug precursors and home-made explosives. The project combines highly advanced disciplines, like organic chemistry, micro fabrication and hardware technology, machine learning and signal processing techniques. It will provide customs officers, police and other authorities with an effective tool to control trafficking of illegal and dangerous chemicals.

The main objective is to develop an outstanding miniaturized, "Sniffer" system based on new colorimetric sensor technology. It should give nearly real-time information about potential illegal substances through detection of their vapor with high specificity and sensitivity, low cost and high energy efficiency and without extensive user training.

Target analytes include illicit drugs and their precursors, commercial and military explosives, improvised explosives and their precursors, and energetic materials in general.

*jmunk@nanotech.dtu.dk; phone +45 4525 8142; www.nanotech.dtu.dk / www.crimtrack.eu

Optics and Photonics for Counterterrorism, Crime Fighting, and Defence XI; and Optical Materials and Biomaterials in Security and Defence Systems Technology XII, D. Burgess, G. Owen, H. Rana, R. Zamboni, F. Kajzar, A. A. Szep, Eds., Proc. of SPIE Vol. 9652, 965208 · C 2015 SPIE · CCC code: 0277-786X/15/\$18 - doi: 10.1117/12.2194915 


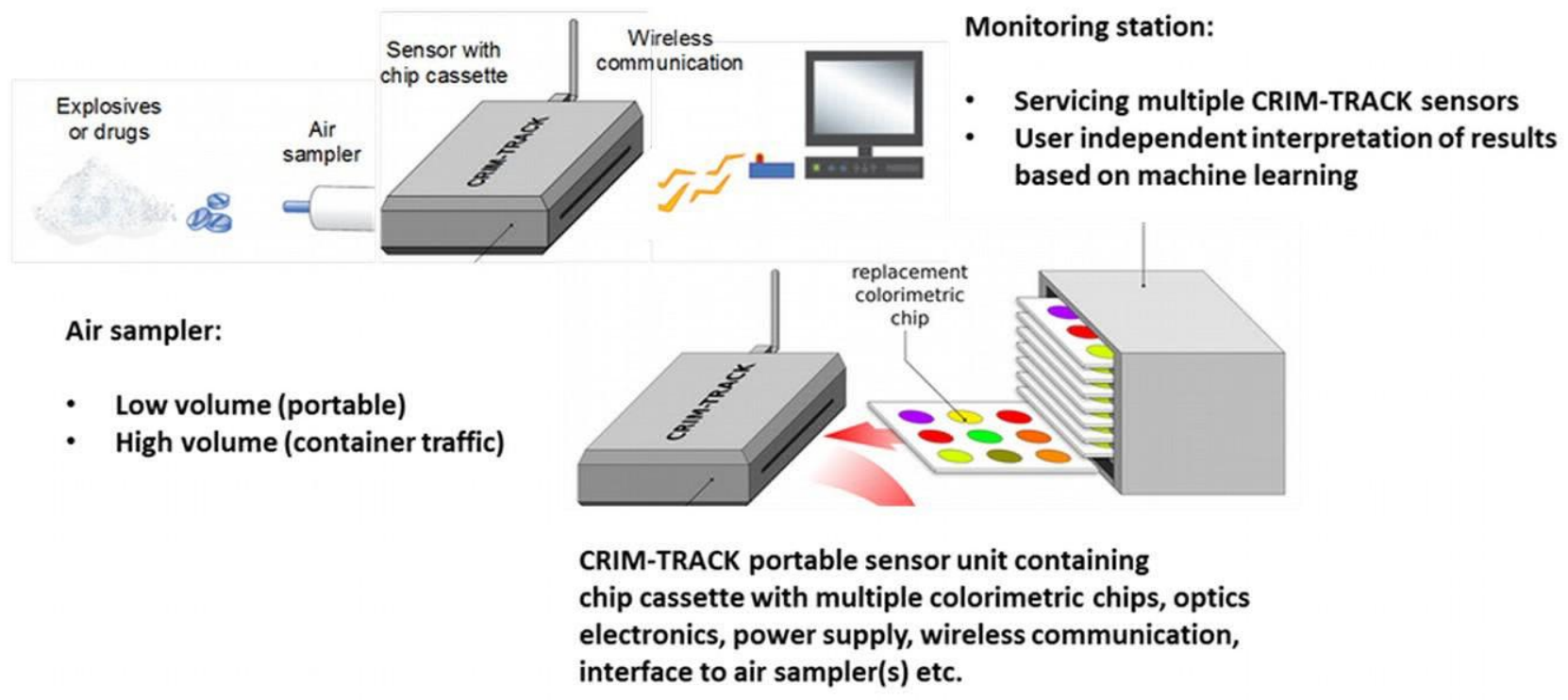

Figure 1: Outline of the CRIM-TRACK "Sniffer" system.

Three prototype versions of "sniffer" systems will be produced and initially validated in controlled environments. Each version will include improved capabilities and also becoming more portable and robust thus, enabling the use at ambient conditions at several test sites. Finally, the system will be validated by law enforcement agencies (LEAs) in three demonstration work packages:

1. Border control - Hidden drugs led by Dutch Customs Laboratory (NL)

2. Checkpoint security - Hidden drugs led by Mykolas Romeris University and the Lithuanian police (LT)

3. Forensic investigation - Explosives led by the Danish Emergency Management Agency (DK)

\section{CURRENT PROJECT STATUS}

At current we have a prototype machine that weighs $\sim 10 \mathrm{~kg}$ and is portable (Figure 1C,D). It is also battery operable and relatively simple to use. It houses the following components:

- Camera

- LED illumination

- Illumination brightness sensor

- Air pump

- Buffer vessel

- Temperature sensor

- Humidity sensor

- Airflow sensor

- Control electronics

- Connective tubing and cabling

- Batteries

Furthermore we have developed a colorimetric sensor chip that is optimized for sensing $\sim 15$ chemicals, identified by our LEA partners as being the most relevant to the project at this stage. Several copies of the same dye are spotted in a randomized 15-by-15 spot pattern. A flow chamber has been developed to produce an airflow that exposes the chip to the analyte vapors in the sample air. 


\subsection{Collecting Analyte Data}

The current procedure to running a session of detection of an analyte in an air sample is as folllows:

1. Chip is loaded into a chip carrier made from soft material (Figure 2A).

2. Chip in carrier is loaded into a flow chamber, which allows connection to standard tubing via blue connectors. Grey clamps ensure air tightness (Figure 2B).

3. Flow chamber is clicked into place on the prototype using magnets, black tubing is connected (Figure 2C).

4. Light blocking hood is clicked in place (Figure 2D).

Tubing can then be connected to allow an air sample to access the chip. Changing a chip takes less than one minute. A computer controlling the machine then runs a script, controlling the machine's hardware components and collecting the data.

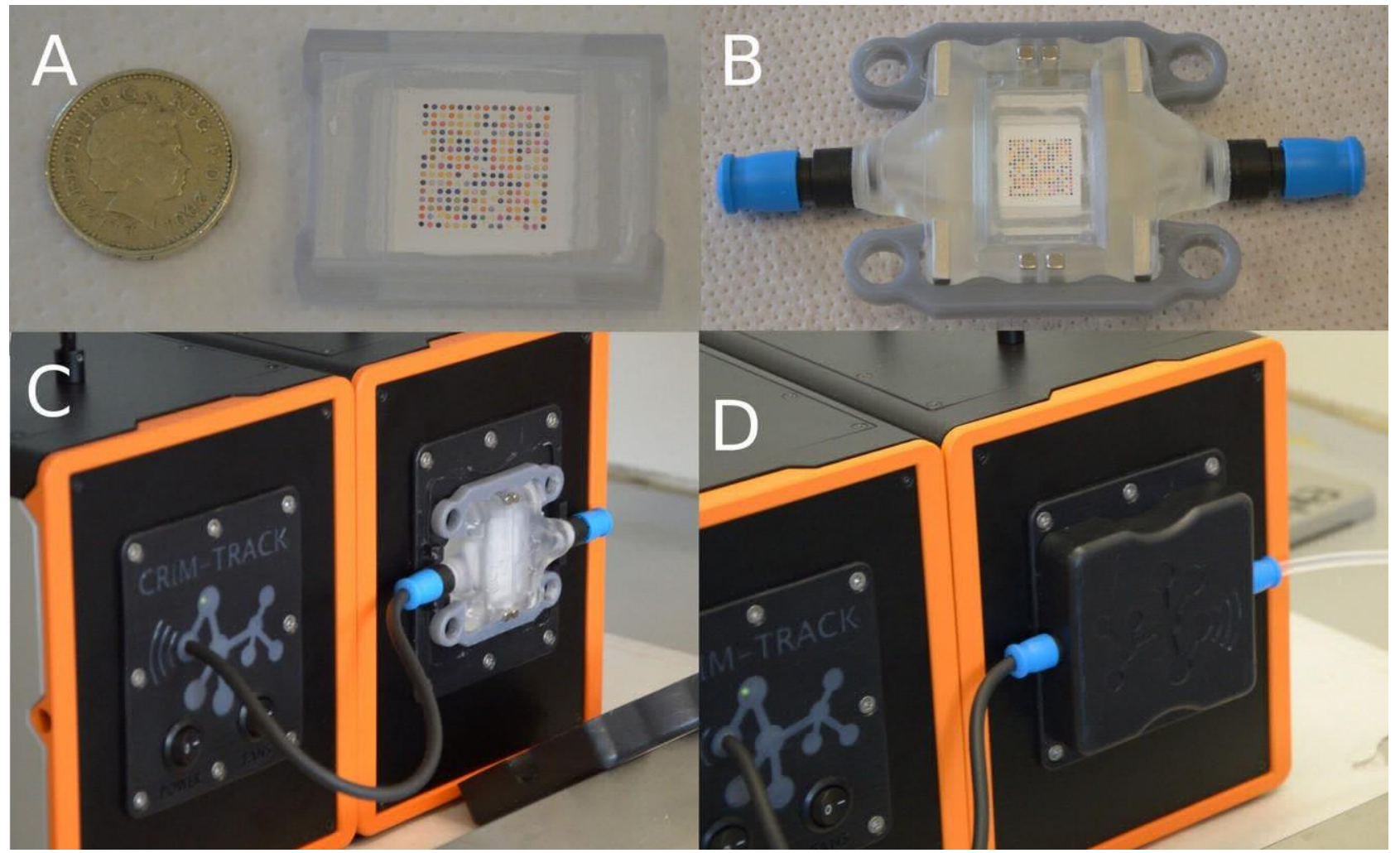

Figure 2: Mounting sensor chip in current prototype. A: Chip in rubber chip carrier, $£ 1$ coin as size reference. B: Chip loaded into flow chamber, grey clamps ensure air tightness. C: Flow chamber clicked into place on prototype, black downstream air tubing connected. D: Black light blocking hood mounted atop flow chamber, clear upstream air sample tubing connected.

\subsection{Performing Data Analysis}

The machine records analyte data as a series of high-resolution pictures taken using an RGB camera. To observe color changes in the spots, we produce difference maps and graphs.

\subsubsection{Difference Maps}

To present signals visually we produce difference maps by simply subtracting a picture taken prior to analyte exposure from a picture taken after some period of time of analyte exposure. Figure 3A shows a difference map for water, Figure 
3B shows a difference map for hydrogen peroxide $30 \%$ in water, which is a precursor of homemade explosives. The clear difference between the maps shows detection of hydrogen peroxide.

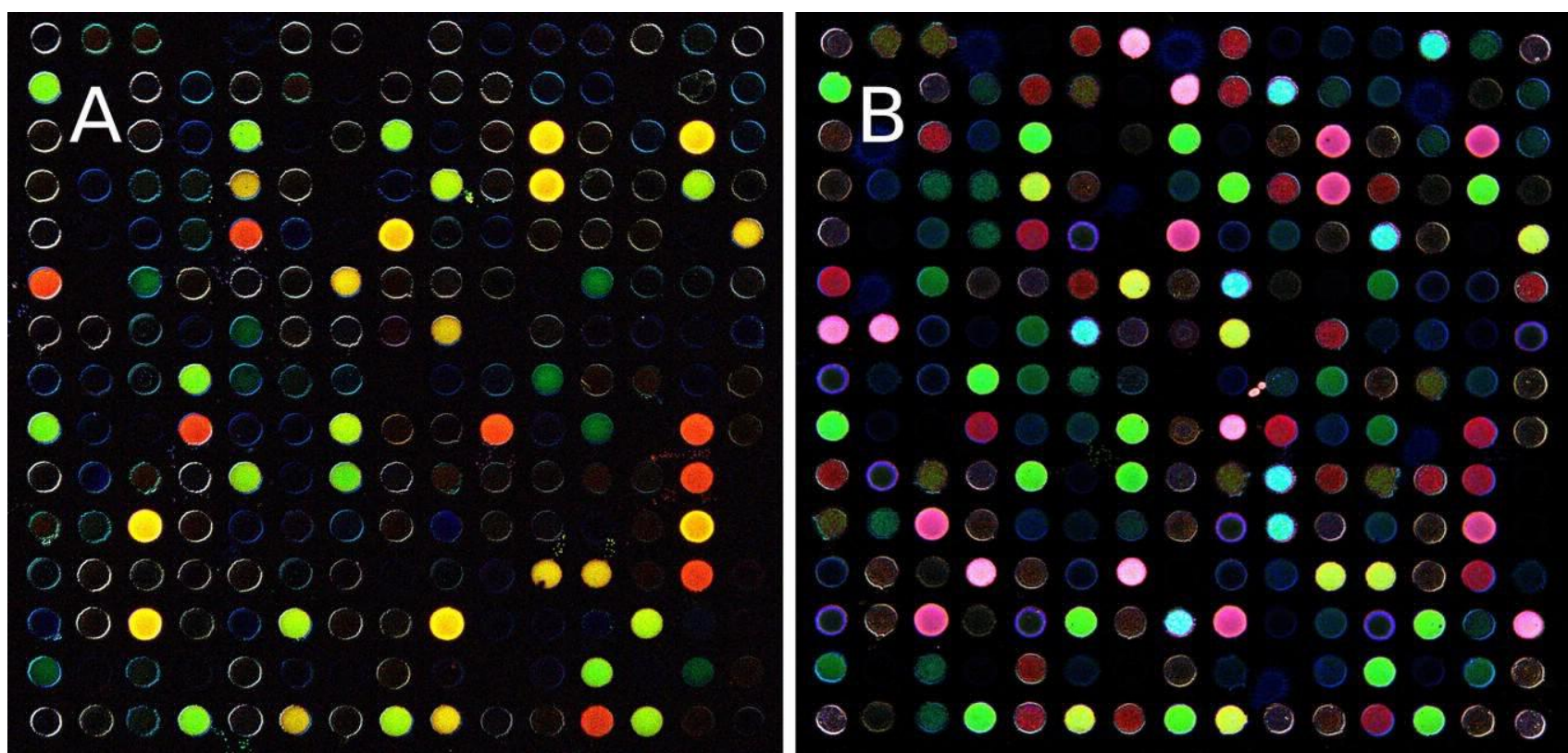

Figure 3: Difference maps showing signals from several dyes. Signals are enhanced for clarity. A: Map of water, showing $\sim 5$ strongly reacting dyes. B: Map of hydrogen peroxide $30 \%$ in water, showing $\sim 10$ strongly reacting dyes.

\subsubsection{Graphs}

The first type of graph is the box plot. To produce a box plot, the spots in the pictures are located with software. The pixel values in these spots are then extracted as numbers, allowing comparison of pixel RGB values before and after exposure of the chip to analyte. Pixel RGB values differences before and after analyte exposure can then be calculated and visualized as box plots. One such is shown in Figure 4A. All spots made from the same dye are combined to show one box plot per dye.

The pixel RGB values can also be used to investigate differences between a series of pictures. We can visualize what happens over time during exposure of a chip to an analyte, focusing on a few dyes that show strong color change. This is shown in Figure 4B.
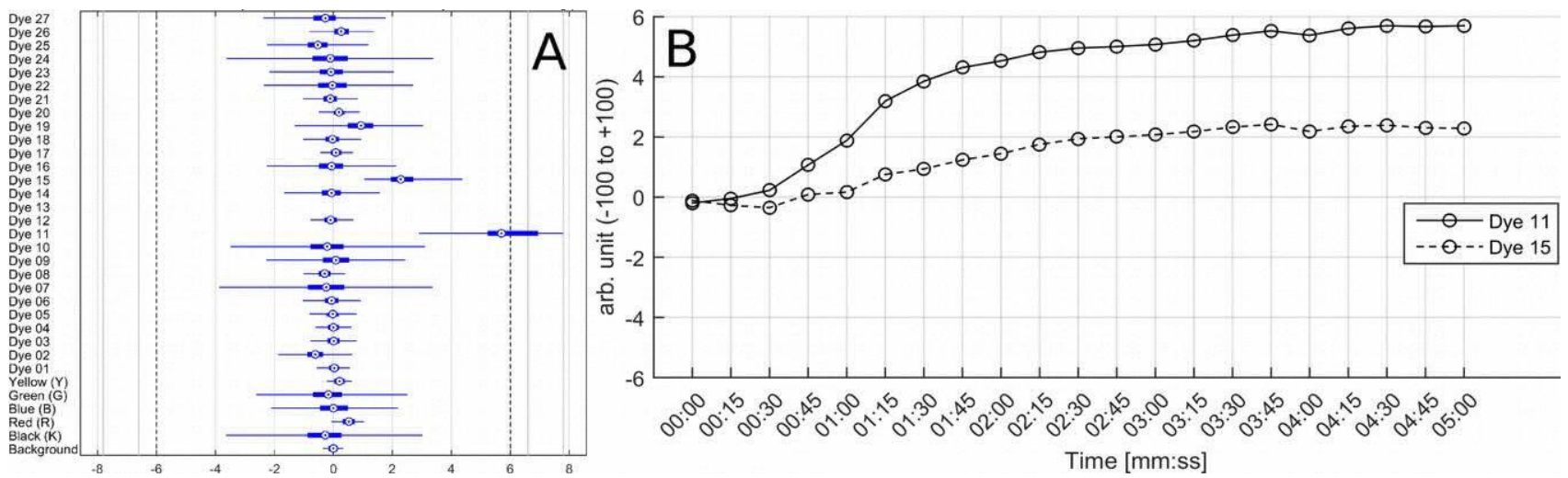

Figure 4: Graphs showing dyes reacting to phenylacetone (drug precursor). A: Box plot representation of all dyes; $\mathrm{x}$ axis unit is arbitrary. B: Evolution of signal from two dyes over time. Signals appear after one minute. 


\section{CONCLUSION AND OUTLOOK}

\subsection{Machine Learning}

The current prototype will be used to produce data which will be used to build a knowledge base on relevant analytes. This data will be used to develop machine learning algorithms capable of detecting the presence of analytes based on how they cause changes in the dye colors.

\subsection{New Prototype}

By mid-2016 we aim to have developed the machine further. The machine should be reduced in size and weight, targeting ease of handling. To accomplish this, the optical equipment will be reduced in size. Also, the chip and flow chamber will be heavily revised to a single disposable unit. This will make chip changing even easier while abolishing the need for user chip handling, a potential cause of error.

\section{ACKNOWLEDGEMENTS}

The CRIM-TRACK project is funded by EU FP7 Grant Agreement Number 313202.

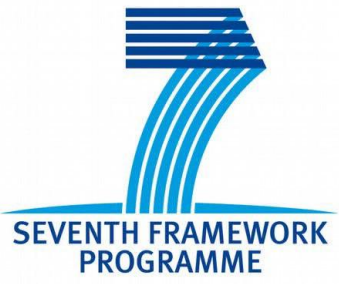

CRIM (") f. TRACK

PROGRAMME 